\title{
Texture evolution in a CrMnFeCoNi high-entropy alloy manufactured by laser powder bed fusion
}

\author{
X. Y. He ${ }^{1}$, H. Wang ${ }^{1, *}$ (1) Z. Z. G. Zhu ${ }^{2}$, L. Z. Wang ${ }^{3}$, J. Q. Liu ${ }^{1}$, N. Haghdadi ${ }^{4}$, S. M. L. Nai ${ }^{2}$, \\ J. Huang ${ }^{3}$, S. Primig ${ }^{4}$, S. P. Ringer ${ }^{1}$, and X. Z. Liao ${ }^{1, *}$ \\ ${ }^{1}$ Australian Centre for Microscopy \& Microanalysis, and School of Aerospace, Mechanical and Mechatronic Engineering, The \\ University of Sydney, Sydney, NSW 2006, Australia \\ ${ }^{2}$ Singapore Institute of Manufacturing Technology, 73 Nanyang Drive, Singapore 637662, Singapore \\ ${ }^{3}$ School of Chemical and Biomolecular Engineering, Sydney Nano Institute, The University of Sydney, Sydney, NSW 2006, Australia \\ ${ }^{4}$ School of Materials Science \& Engineering, UNSW, Sydney, NSW 2052, Australia
}

Received: 17 September 2021

Accepted: 12 February 2022

Published online:

4 March 2022

(C) The Author(s) 2022

\begin{abstract}
Additive manufacturing (AM) techniques including laser powder bed fusion have been widely used to produce metallic components with microstructures and mechanical properties distinctly different from the conventionally manufactured counterparts. Understanding how AM parameters affect the evolution of microstructure, including texture, of these AM metallic components is critical for appropriate manipulation of their processing and therefore their mechanical properties. Here we conducted a systematic investigation of texture evolution of a face-centred cubic $\mathrm{CrMnFeCoNi}$ high-entropy alloy cuboid fabricated using laser powder bed fusion. Our results showed that the texture evolutions along the build direction were different between the corner and central parts of the sample. Detailed analysis suggested that the texture evolution is closely related to local thermal gradient, which is a property that can be manipulated through changing AM parameters. The different textures lead to the significant variations of mechanical properties within the sample.
\end{abstract}

\section{Introduction}

Additive manufacturing (AM) is an advanced technology for rapid production of components with complex geometries. Opposed to the conventional subtractive manufacturing approaches where unwanted materials are removed from an over- dimensioned bulk piece, AM is an incremental process based on layer-by-layer deposition of materials from a feedstock of wires or powders that is often selectively melted by a high power focused laser or electron beam [1-4]. As a near-net or net shape fabrication technology, $\mathrm{AM}$ significantly reduces the materials cost by only consuming the required amount of feedstock materials and therefore is ideal

Handling Editor: M. Grant Norton.

Address correspondence to E-mail: hao.wang1@sydney.edu.au; xiaozhou.liao@sydney.edu.au 
for producing components made from expensive materials like many high-entropy alloys (HEAs) [5]. Structural components fabricated by AM can achieve superior properties [6-10], and this is related to the unique microstructures produced by AM [11, 12]. For example, a previous research suggested that AM $\mathrm{CrMnFeCoNi}$ samples exhibit improved yield strength and ultimate tensile strength compared with as-cast and recrystallized samples fabricated using conventional methods, while maintaining reasonable ductility good enough for most applications [13].

Laser powder bed fusion (LPBF) is one of the most widely used AM techniques with significant industrial applications $[1,3,4]$. During the LPBF process, metallic powders are melted by a high power focused laser beam to fabricate net-shaped or near-net-shaped products [4]. The processing parameters such as scanning speed and laser power are controlled by computer programs [3]. Successive layers of materials are formed by solidification of metal melt [1]. The layer-by-layer materials deposition and traverse scanning path during the LPBF process significantly influence the solidification texture owing to the local thermal gradients and preferred grain growth direction [14-18], which is $<001>$ for face-centred cubic (FCC) alloys [19-22].

Crystallographic texture is an important microstructural characteristic that impacts significantly on mechanical properties and corrosion behaviour of metallic materials [20, 21, 23]. Therefore, the control of crystallographic texture and understanding its evolution are critical for producing AM components with desired mechanical and corrosion properties. While texture control or alternation can be achieved by adopting different scanning strategies [19-21, 24-26], different parts of a complex AM component could still present different textures. Therefore, an in-depth study of the texture evolution at different locations within individual components manufactured by identical processing parameters is necessary. This allows the understanding of the mechanism of texture development, which will guide processing parameters setting and procedure design to produce materials with desired crystallographic textures.

The crystallographic texture of an AM component is strongly affected by local temperature gradient at the growth interface [27] and solidification growth rate $[16,17,28]$, which can be tailored via controlling processing parameters including hatch distance, power, scanning speed, and scanning strategy $[14,16,28,29]$. There have been several reports on the texture formation and evolution during laser assisted AM in alloys with FCC [19-21], body-centred cubic [24], hexagonal close-packed [25], and tetragonal [26] structures. However, the mechanism for texture evolution within a single product manufactured by identical processing parameters is not yet well understood. Here, we used a $\mathrm{CrMnFeCoNi} \mathrm{HEA} \mathrm{with}$ an FCC structure, also known as the Cantor alloy, manufactured by LPBF as a sample material to explore the mechanisms of texture evolution determined by local thermal gradients. The Cantor alloy has exceptional mechanical properties at both ambient and cryogenic temperatures [12, 30]. A combination of high strength and excellent ductility can be achieved by LPBF [13]. We analysed the crystallographic texture evolution using the scanning electron microscopy electron backscatter diffraction (SEMEBSD) technique. Distinct texture evolutions were revealed at the centre and corners of an as-fabricated cubic component. The crystallographic texture is consistent in the central part of the sample. However, gradual transition of texture orientation along the build (z) direction was observed in the corner parts. These texture differences lead to significant variations of mechanical properties within the sample. The mechanism behind this texture development and how the texture impacts the mechanical properties are discussed.

\section{Methodology}

A CrMnFeCoNi HEA was fabricated using LPBF in the shape of a cube with an edge length of $10 \mathrm{~mm}$. The alloying powder used for AM was produced by gas atomisation [13]. The LPBF process was performed by a ProX 300 laser assisted ( $500 \mathrm{~W}$ per fibre) powder bed fusion system (3D) with the following processing parameters: $60 \mathrm{Jmm}^{-3}$ energy density, $2000 \mathrm{~mm} \mathrm{~s}^{-1}$ scanning speed, $240 \mathrm{~W}$ laser power, $50 \mu \mathrm{m}$ hatching space, $40 \mu \mathrm{m}$ layer thickness, and $75 \mu \mathrm{m}$ laser beam diameter [8]. Bi-directional linear scans in each layer with the scanning angle variation between successive layers of $90^{\circ}$ was adopted [13]. The scan directions were approximately $45^{\circ}$ deviated from the $x$ - and $y$-axes of the sample around the build direction [13]. The composition of the as-built CrMnFeCoNi cubic sample, which was measured 
using instrumental gas analysis and inductively coupled plasma mass spectroscopy for non-metallic light elements and metallic elements, respectively, is 19.3Cr-19.6Mn-20.0Fe-20.6Co-20.3Ni-0.050O-0.017 N$0.0011 \mathrm{H}$ in atomic percentage [13].

For microstructure observation in the $x-z$ plane (see Fig. 1a for the definition of $x, y$, and $z$ axes), the as-built HEA was sliced into cuboids with dimensions of $3 \times 3 \times 10 \mathrm{~mm}^{3}$ along the $z$-axis (build direction) using an Accutom-50 diamond saw and was then polished by $0.05 \mu \mathrm{m}$ oxide dispersion suspension [11]. To reveal the boundaries of melt pools, the samples were etched for $30 \mathrm{~s}$ in the solution where the ratio of solute and solvents was $\mathrm{HNO}_{3}$ : $\mathrm{HCl}: \mathrm{H}_{2} \mathrm{O}=1: 3: 2$. The melt pool morphology was examined using a Leica DMi8M optical microscope $(\mathrm{OM})$ under the polarised mode. The microstructure observation was performed using Zeiss Ultra SEMEBSD at an accelerating voltage of $20 \mathrm{kV}$ and a step size of $50 \mu \mathrm{m}$. The obtained data were processed using the AZtecHKL software.

Vickers micro-indentation tests were performed using a LECO Vickers harness tester (LV700AT). Hardness was measured at a load of $0.3 \mathrm{kgf}$ and dwell time of $15 \mathrm{~s}$. A spacing of at least $200 \mu \mathrm{m}$ was applied between neighbouring indentations to eliminate cross-interactions between indented volumes.

\section{Results}

The near-fully dense ( $>99.2 \%$ as measured by the Archimedes method) product with an FCC structure was obtained. The as-built sample was divided into corner (grey) and centre (blue) parts perpendicular to the $x-y$ plane with nearly identical geometries as illustrated in Fig. 1a. The build direction is denoted as the $\mathrm{z}$ direction in Fig. 1a. Figure $1 \mathrm{~b}$ presents $\mathrm{a}$ schematic diagram marking regions of top, middle, and bottom in each sample. The OM image in Fig. 1c taken on the $\mathrm{x}-\mathrm{z}$ plane revealed the trace of melt pools (tracked by yellow dash lines) and the morphology of columnar grains. The melt pool geometry on the $\mathrm{x}-\mathrm{z}$ plane was in a shape of a flattened half ellipse elongated along the horizontal direction with the major axis around $100 \mu \mathrm{m}$ compared to the layer thickness of $40 \mu \mathrm{m}$. The columnar grains tended to develop along the build ( $\mathrm{z}$ ) direction and extended across several melt pool boundaries (an example is presented in Fig. 1d), indicating the epitaxial growth nature of these grains. These columnar grains aligned along the build direction facilitated the establishment of strong textures, which will be further elaborated when presenting EBSD observations later.

Figure 2 presents EBSD inverse pole figure (IPF) maps along the z-axis (IPF-z) and the y-axis (IPF-y) showing crystallographic textures of the central part of the sample in the bottom, middle and top regions. The IPF-z maps in Fig. 2a-c, mostly red, revealed the intense alignment of $<001>$ texture with the build (z) direction in the bottom, middle, and top regions. While the IPF-y maps in Fig. 2d-e, predominantly green, indicated the close alignment of $<101>$ texture with the $x$ - and $y$-axes in the bottom, middle, and top regions. It is noticed that the grains are highly elongated towards the build direction in Fig. 2. Slightly radical extensions are also noticed for the elongated grains, which is attributed to the local
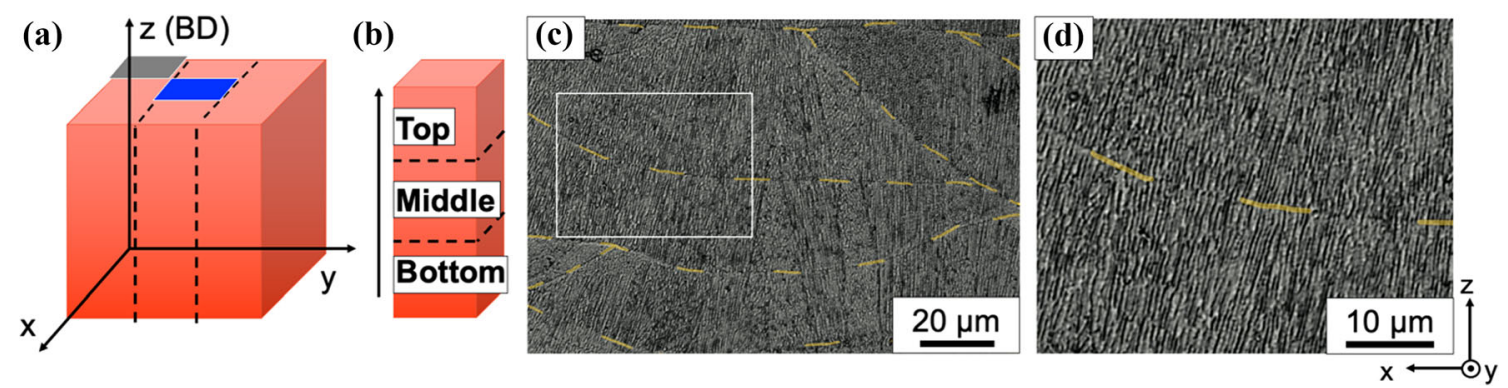

Figure 1 a Schematic of the fabricated cubic product and the locations of corner and centre samples. The build direction is denoted by BD. The centre and corner locations are indicated by blue and grey colours, respectively. $\mathbf{b}$ Schematic diagram showing positions of top, middle, and bottom regions in each sample. The arrow represents the build direction. c A typical OM image taken on the $\mathrm{x}-\mathrm{z}$ plane showing surface morphology, including columnar grains and melt pools. The yellow dash lines indicate melt pool boundaries. d An enlarged OM image at the rectangular local region in (c), displaying epitaxial grain growth across the melt pool boundary. 
Figure 2 EBSD IPF maps from the central part of the sample. a-c IPF-z maps from the bottom, middle, and top regions, respectively. $\mathbf{d}-\mathbf{f}$ IPFy maps from the bottom, middle, and top regions, respectively. All maps were generated on the $\mathrm{x}-\mathrm{Z}$ plane. Grain boundaries are marked by the black lines.
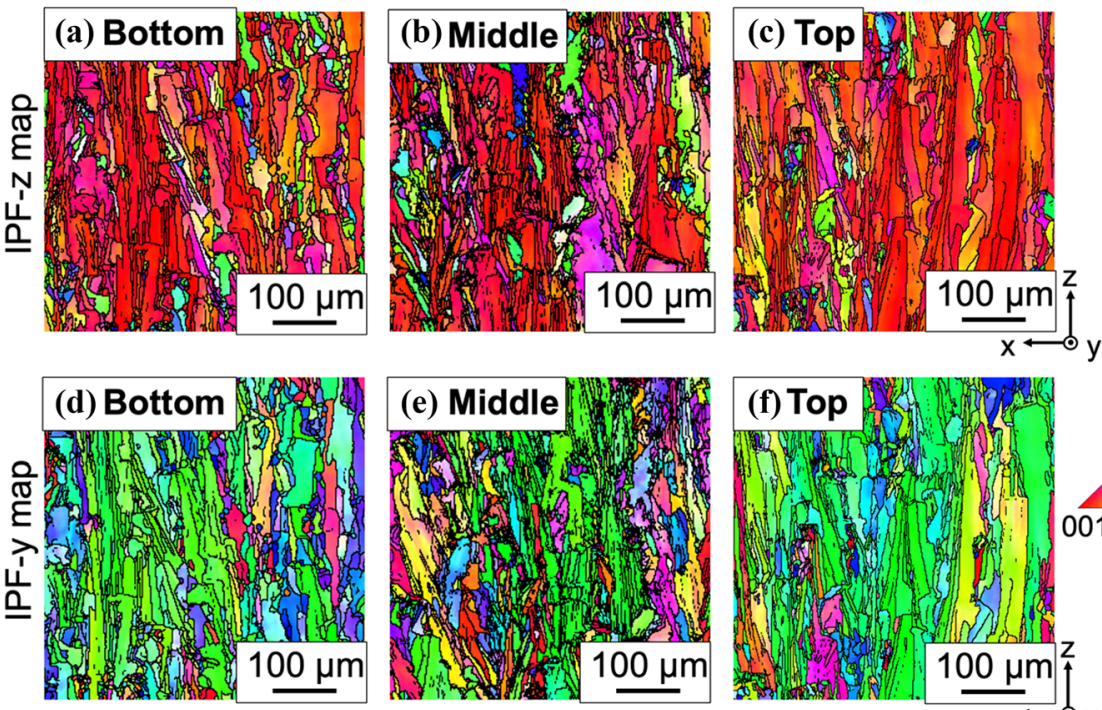

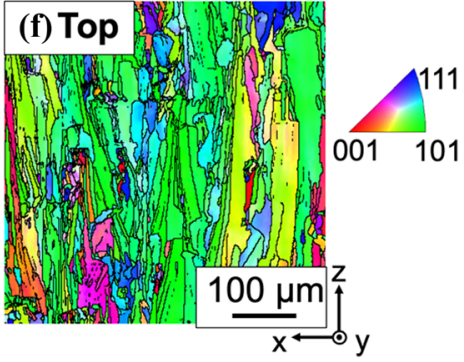

heat flows normal to the melt pool boundaries $[15,26,31]$ affecting the near-vertical development of epitaxial grains.

The corresponding $<001>$ and $<101>$ pole figures for the central part of the sample measured in $x-$ $z$ plane are shown in Fig. 3. One $<001>$ pole remained in line with the build $(\mathrm{z})$ direction as illustrated in Fig. 3a-c, while the remaining $<001>$ poles persisted a misalignment of $45^{\circ}$ with respect to the $x$ - and $y$-axes throughout all the regions. Figures $3 \mathrm{~d}-\mathrm{f}$ suggest strong alignment between poles of $<101>$ and the $x$ - and y-axes in all the regions. These implied that a single-crystalline- like texture was established in the central part of the sample.

Figure 4 presents EBSD IPF maps of a corner part of the sample showing the crystallographic texture in the bottom, middle, and top regions. The IPF-z maps in Fig. 4a-c, largely red, revealed the close match of $<001>$ texture with the build $(\mathrm{z})$ direction in the bottom, middle, and top regions. The IPF-y maps in Fig. $4 \mathrm{~d}$, predominantly green, indicated strong alignment of $<101>$ texture with the $y$-axis in the bottom region. Figure $4 \mathrm{e}$ illustrates a combination of $<001>$ and $<101>$ crystallographic orientation along the $y$-axis in the middle part with a mix of green and red colours. Figure $4 \mathrm{f}$, mostly red, shows
Figure. 3 EBSD pole figures from the central part of the sample. $\mathbf{a}-\mathbf{c}<001>$ pole figures from the bottom, middle, and top regions, respectively. d-

$\mathbf{f}<101>$ pole figures taken from the bottom, middle, and top regions, respectively.

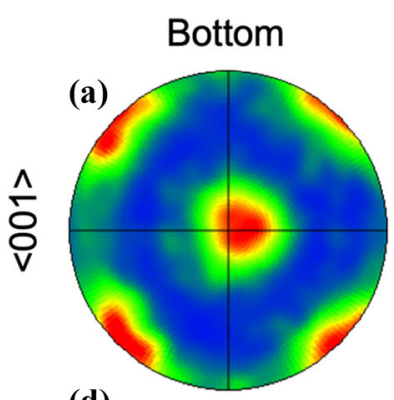

(d)
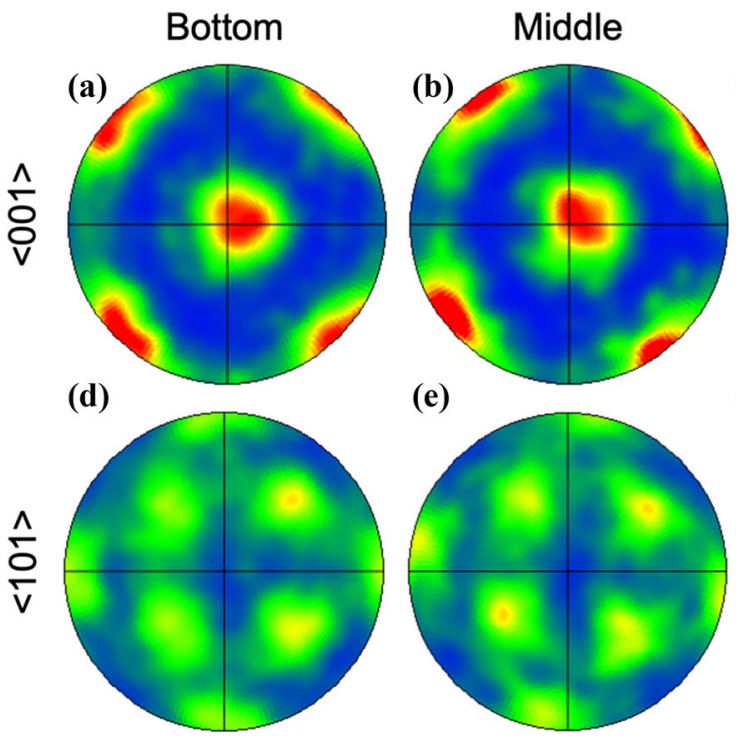

(e)

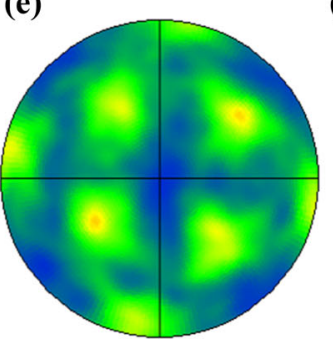

(f)
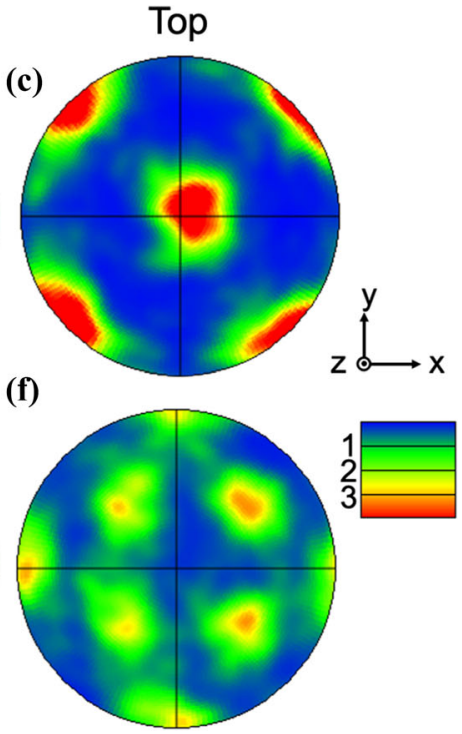
Figure 4 EBSD IPF maps from the corner part of the sample. a-c IPF-z maps from the bottom, middle, and top regions, respectively. $\mathbf{d}-\mathbf{f}$ IPFy maps from the bottom, middle, and top regions, respectively. All maps were generated on the $\mathrm{x}-\mathrm{Z}$ plane. Grain boundaries are marked by the black lines.
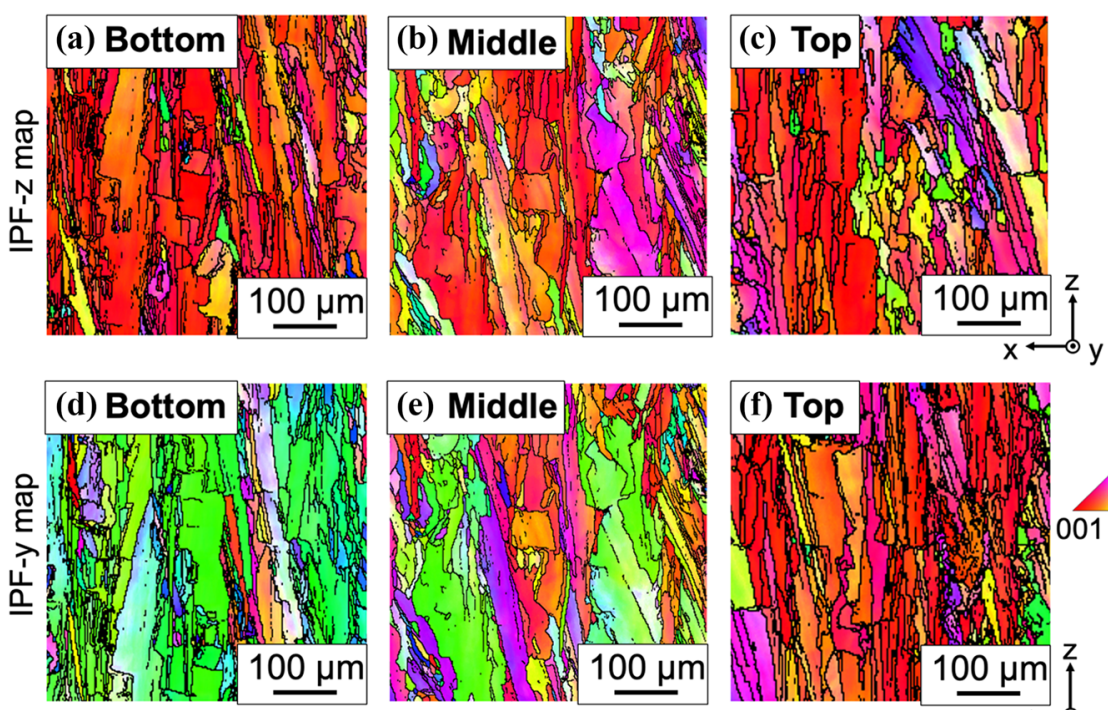
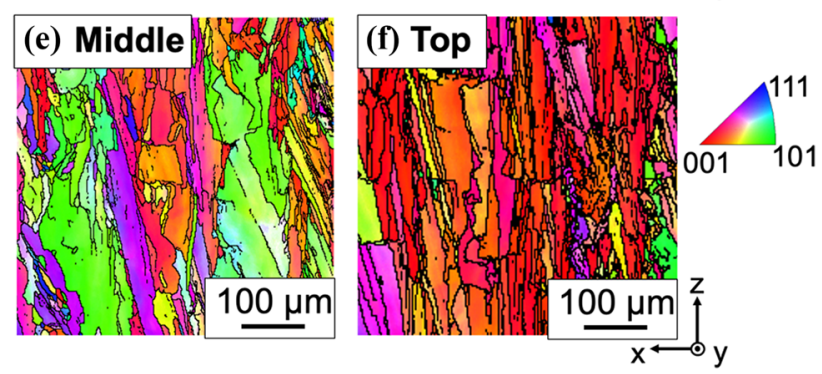

intense alignment of $<001>$ texture with the $y$-axis in the top region.

The corresponding $<001>$ and $<101>$ pole figures for the corner part of the sample are shown in Fig. 5. One of the $<001>$ poles was in line with the build ( $\mathrm{z}$ ) direction as illustrated in Fig. 5a, while the remaining poles of $<001>$ persisted a misalignment of $45^{\circ}$ with respect to the $\mathrm{x}$ - and $\mathrm{y}$-axes in the bottom region. The $<001>$ poles began to rotate clockwise around the $\mathrm{z}$-axis in the middle region as demonstrated in Fig. $5 b$, where one of the $<001>$ poles remained in alignment with the build direction. In Fig. $5 c$, the $<001>$ poles appeared to have displaced approximately $45^{\circ}$ clockwise around the z-axis in the top region compared to that in the bottom region, where all the $<001>$ poles aligned with the three principal axes in the top region. While Fig. $5 d$ suggests strong alignment between poles of $<101>$ and the $x$ - and $y$-axes in the bottom region, Fig. 5e-f illustrates no sharp concentration of $<101>$ poles along any of the axis in the middle and top regions. It was also noticed that the rotational displacement of the $<101>$ poles was consistent with what was observed in the $<001>$ pole figures. The $<101>$ poles began to rotate clockwise around the z-axis in the middle region as demonstrated in Fig. 5e. The $<101>$ poles appeared to have moved approximately $45^{\circ}$ clockwise around the z-axis in the top region (Fig. 5f) compared to that in the bottom region (Fig. 5d).
Figure 5 EBSD pole

figures from the corner parts of the sample. $\mathbf{a}-\mathbf{c}<001>$ pole figures from the bottom, middle, and top regions, respectively. $\mathbf{d}-$

f $<101>$ pole figures from the bottom, middle, and top regions, respectively.
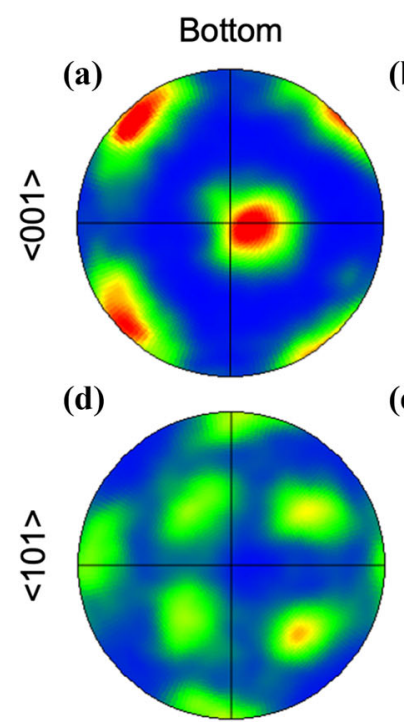

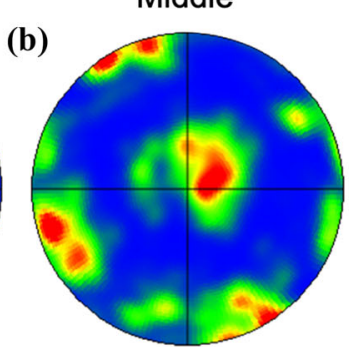

(e)

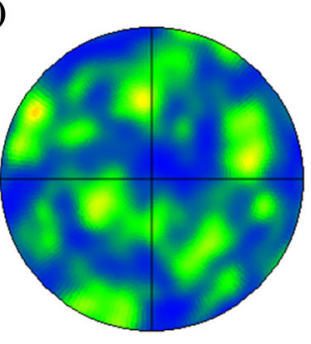

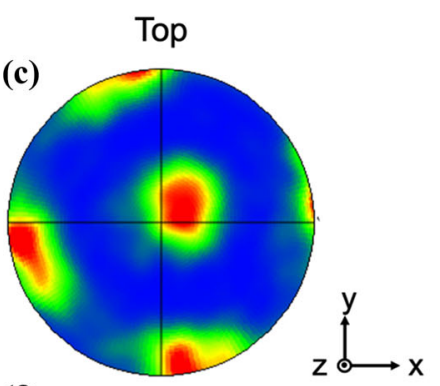

(f)

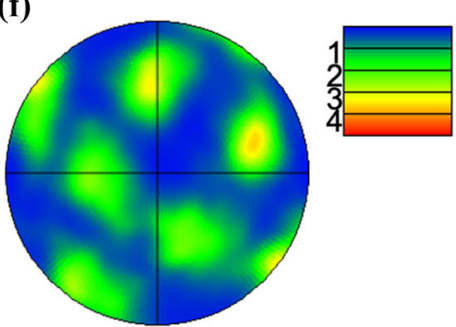


Different mechanisms of texture evolution were in place throughout the as-built sample at different locations. At the central part of the sample, strong $<101>$ alignment along the $x$ - and $y$-axes was observed, while the preferred growth direction along the build ( $\mathrm{z}$ ) direction was $<001>$. The texture remained the same along the build direction from the bottom to the top of the sample. On the other hand, while a sharp intensity of $<001>$ in the build direction was noticed in all three regions, the corner part of the sample exhibited gradual transformation in texture orientations. A transition from striking $<101>$ to intense $<001>$ texture parallel to the $\mathrm{x}$ - and $\mathrm{y}$-axes was observed across each region.

Different texture evolutions at the central and corner parts of the sample contribute to distinct local mechanical properties. Figure 6 visualizes the microhardness for the top, middle, and bottom regions ( $\mathrm{x}-\mathrm{z}$ cross section) at the central (the left column) and corner (the right column) parts of the asbuilt sample. While the hardness at the central part of the sample is relatively homogeneous at around 220 $\mathrm{HV}$, the hardness at the corner part of the sample presents considerable increase ranging from $240 \mathrm{HV}$ at the bottom to $320 \mathrm{HV}$ at the top. The local hardness at the corners is more inhomogeneous and much higher than that at the centre.

\section{Discussion}

It is generally recognised that the development of crystallographic texture in AM components is a consequence of the cooperated effects of the direction of thermal gradient $[14,15,17,18,22,27,31]$ and the

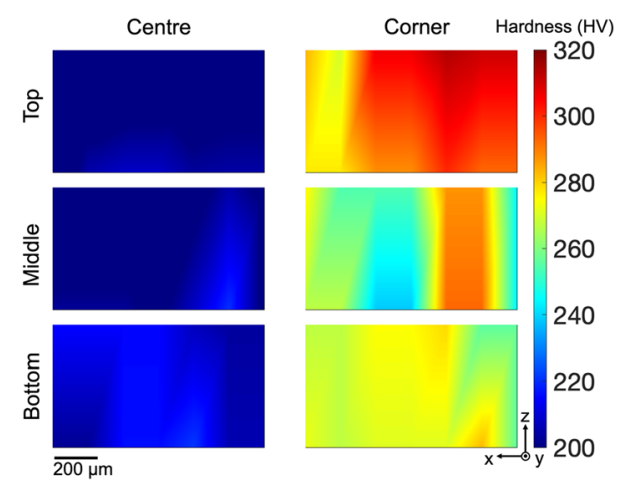

Figure 6 Two-dimensional hardness maps from top, middle, and bottom regions of the central and corner parts of the sample. The outer end of the corner is at the right. decreased nucleation energy enabled by epitaxial growth [15, 32, 33], the cooling rate [16, 17, 28], and the scan strategy [19-21, 24-26]. In particular, the crystallographic growth in $<001>$ directions should occur along the directions with large thermal gradients $[15,22,34]$ and the crystallization growth rate of the crystals increases monotonically with the magnitude of thermal gradient [15, 17]. Previous studies suggest that texture control is deemed to be successful upon the initial selection of preferentially growth of columnar grains. The control at the initial stages is dependent on the scan strategy and the consistent texture development is assisted by epitaxial growth [19, 24, 34]. However, this is obviously different from the observation in Figs. 4 and 5, where the texture can still experience changes in the middle and top regions after being initially determined by the scan strategy in the bottom region. Therefore, the proposed factors affecting texture evolution and a more detailed mechanism within a single sample need to be evaluated.

In both centre and corner sample locations, a vertical $<001>$ texture forms parallel to the build direction as similar to other body-centred cubic and FCC metals fabricated by xy-scan [19, 21, 24, 26]. This growth behaviour is stimulated by the propensity to minimize the nucleation energy by preserving the crystallographic orientation between melt pools in the subsequent layers where the scan direction is switched by $90^{\circ}$ [24]. The principal resolution to reduce the angle between the direction of epitaxial growth of the underlying columnar grains and the thermal gradient direction in the newly deposited layer is acquired by the grain growth parallel to the build direction [24]. This is also consistent with the previous observation that $<001\rangle$ is the easiest growth direction that usually aligns with the maximal heat flow direction for FCC metals [19-21, 34]. Here, a more in-depth clarification is required to address the detailed mechanism for texture (along the $x$ - and y-axes) transition across different locations within the product.

To better explain the contributing factors to the texture evolution, schematics of the distribution of thermal gradients on the $\mathrm{x}-\mathrm{y}$ plane in the bottom, middle, and top regions are presented in Fig. 7. A schematic for the scan strategy is also illustrated in Fig. $7 d$, which corresponds to the thermal gradient directions at the bottom region. In the central part of the sample, the crystallographic orientations remain 
the same along the build direction from the bottom to the top. The $<101>$ texture is favourably aligned with the $x$ - and y-axes, resulting in a misalignment of $45^{\circ}$ between the preferred growth directions $<001>$ and the $x$ - and $y$-axes throughout all regions. This misalignment is closely associated with the scan strategy. This is because the cooling rate is the product of the scan speed and thermal gradient $[14,15,17]$, and the cooling rate is extremely high near the substrate at the bottom part of the sample $[1,14,31]$. The largest thermal gradients on the $x-y$ plane are always aligned with the scan directions which are approximately $45^{\circ}$ deviated from the $x-$ and $y$-axes of the sample around the build direction as illustrated in Fig. 7a. The angle between the directions of $<001>$ and $<101>$ on the horizontal plane is also $45^{\circ}$, which is consistent with the prediction that the $<001>$ texture is favourably developed parallel to the scan directions in bi-directional scanning [19, 24, 34]. This texture arrangement conserves the crystallographic orientation for epitaxial growth between two neighbouring scan paths on the horizontal plane [34]. In the middle and top regions of the central part of the sample, the cooling rate decreases due to heat accumulation along the build direction $[1,14]$. Here, the maximal thermal gradients that determine the $<001>$ growth directions on the $\mathrm{x}-\mathrm{y}$ plane remain being governed by the scan directions as demonstrated in Fig. $7 \mathrm{~b}$ and $\mathrm{c}$. There is no other contribution that could possibly deviate the direction of the thermal gradients exerted by the scan strategy. Therefore, identical crystallographic textures form along the build direction from the bottom to the top in the central part of the sample.

The texture development appears to be assisted by inter-layer epitaxial growth as shown in the results.
Here, the previously deposited underlying materials are partially remelted by generating the melt pool and the crystallographic orientations of the newly solidified materials developed in accordance with the underlying orientations [14-16, 28]. However, the texture transition across the bottom, middle and top regions occurred in the corner parts of the sample seems to be contradictory to the typical behaviour of epitaxy. Focusing on this feature, the detailed evolution mechanism of the texture is discussed below.

For the corner parts of the sample, the thermal gradients established within melt pools are disturbed by the ambient temperature field adjacent to them. Initially at the bottom, the crystallographic texture shown in Fig. 4a and d is analogous to those observed for the central part of the sample in Fig. 2. This is determined by the combined effects of the initial preferential growth directions being governed by scan strategy $[19,24]$ and the rapid solidification rate near the substrate $[1,14]$. Similar to what has been discussed for the central part of the sample, in Fig. 7a, the extremely high cooling rate near the substrate results in higher thermal gradients along the scan directions on the horizontal plane in the bottom region of the corner areas of the sample. With further fabrication, the corner parts of the sample have direct contact or are adjacent to the ambient chamber environment, where the temperatures are dramatically lower than that within the fabricated product. On the other hand, the central part of the sample is surrounded by the internal environment of the laser heated deposition where the temperatures are supposed to be much higher than the ambient chamber temperature [1]. Since the temperature gradient field surrounding the central part of the sample is reasonably symmetric on the $x-y$ plane, the (a) Bottom

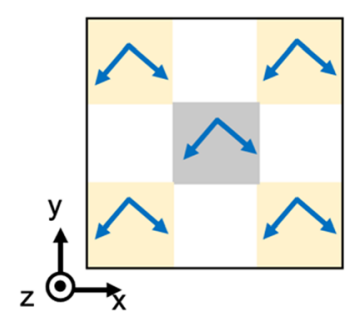

(b) Middle

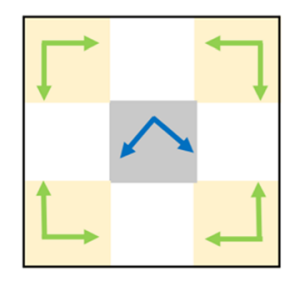

(c) Top

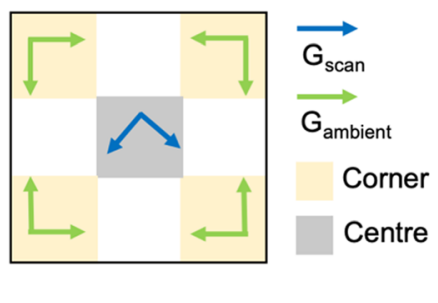

(d)

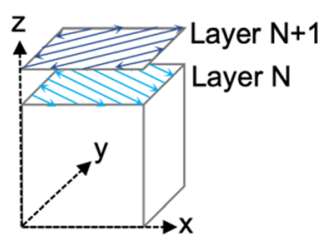

Figure 7 Schematics of thermal gradients on the $x-y$ plane in the a bottom, $\mathbf{b}$ middle, and $\mathbf{c}$ top regions of the sample. The colour pale yellow marks corner parts and the colour grey marks the central part of the sample. Blue arrows represent the largest thermal gradients caused by scan directions and green arrows indicate the largest thermal gradients effected by the ambient temperature. d Schematic of the scan strategy. Build direction is along the z-axis. 
solidification texture is predominantly controlled by the track-by-track scan paths, the $<001>$ texture lies parallel to the scan directions [19, 24, 34]. The asymmetric temperature field on the $x-y$ plane encompassing the corner parts of the sample promotes the highly directional temperature gradients pointing inwards. Further, they are parallel to the $x-$ and y-axes as illustrated in Fig. $7 b$ and c, because the shortest route for the fastest heat dissipation out from the sample on the $x-y$ plane is along the $x$ - and $y$-axes where the material thickness is minimal. The disparity of thermal gradients between these two types of location is created. The cooling rate decreases from the bottom to the top due to thermal accumulation $[1,14]$. Therefore, the thermal gradients along the scan direction are also decreased, and the thermal gradients affected by the ambient temperature start to govern the texture development at the corners. As a result, in the middle and top regions of the corner parts of the sample, the maximal thermal gradients along the $x$ - and $y$-axes far exceed those along the scan paths on the horizontal plane.

Figure 8 illustrates schematically the crucial epitaxial growth process behind the gradual texture evolution in the corner parts of the sample, where similar processes are experienced by all four corners of the sample. These increased temperature gradients towards the $x$ - and $y$-axes coincided with a slower cooling rate in the middle and top regions. This lead to the epitaxial growth of grains with $<001>$ (viewed along the y-axis) orientation being favoured, and subsequent growth of grains with $<101>$ (viewed along the y-axis) orientation being suppressed as illustrated in Fig. 8. The influence from the ambient temperature appears to surpass the impact of scan direction on determination of the preferential $<001>$ growth direction parallel to the horizontal $x-y$ plane as the region of interest approaches the top surface. Being closer to the substrate, the highest cooling rates occur in the bottom region. Therefore, a progressive decline of cooling rate is expected from the bottom to the top regions due to thermal accumulation along the build direction during the layerby-layer manufacturing process $[1,14]$. Therefore, the directions of the highest thermal gradients alter, which affects preferential grain growth and hence texture development. Figure 8a demonstrates the dominant $<101>$ columnar grain viewed along the $y$-axis together with initial nucleation of small $<001>$ grain occurred in the bottom region of the corner part of the sample. This is facilitated by the highest thermal gradients along the scan paths. In the middle region as demonstrated in Fig. 8b, the combined effects of slower cooling rate and enhanced thermal gradient along the $\mathrm{x}$ - and $\mathrm{y}$-axes assist the competitive epitaxial growth of $<001>$ (viewed along the y-axis) columnar grains. While the growth velocity of $<101>$ ( viewed along the $y$-axis) grains decreases since the $<001>$ directions of these grains no longer align with the maximal thermal gradients on the $x-y$ plane as shown in Fig. 7b. Consequently, substantial volumes of both crystallographic orientations are developed in the middle region. As cooling rates further decline in the top region, the temperature gradients along the scan directions are further weakened while the thermal gradients along the $\mathrm{x}$ - and $\mathrm{y}$-axes remain the most significant on the horizontal plane as shown in Fig. 7c. Therefore, the epitaxial growth of the $<$ $001>$ (viewed along the y-axis) columnar grains is favoured and develops due to its preferred growth direction $<001>$ being aligned with the intensified temperature gradient along the $x$ - and $y$-axes. On the contrary, the growth of $<101>$ (viewed along the $y$-axis) grains is severely supressed, as illustrated in Fig. 8c. Here, the impacts from the escalated thermal gradients induced by low ambient temperature and (a) Bottom

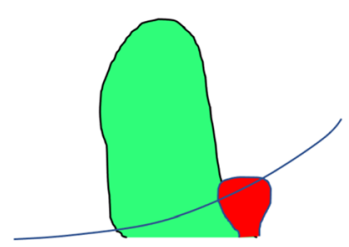

(b) Middle

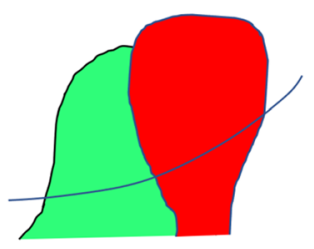

(c) Top

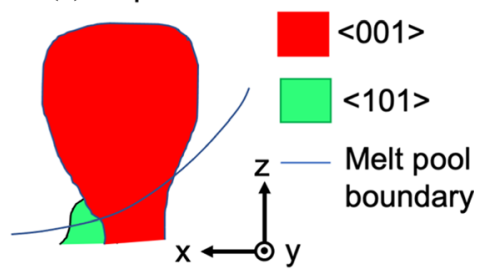

Figure 8 Schematics of epitaxial growth of the $<001>$ and $<101>$ (viewed along the y-axis) columnar grains across the melt pool boundary for the corner parts of the sample at the a bottom, $\mathbf{b}$ middle, and $\mathbf{c}$ top regions. All four corners of the sample experience similar epitaxial growth processes. Areas depicted are not in proportion. 
the alleviated cooling rate are deemed to surpass the domination of the thermal gradients along scan paths on determination of the preferential $<001>$ growth directions parallel to the horizontal $x-y$ plane.

Epitaxial growth is one of the most significant factors in texture evolution [17, 19, 21, 24, 32]. The change in the texture across regions in the corner parts of the sample is not thought to be associated with epitaxy initially due to the alternation of crystallographic texture from bottom to top and its inconsistency across different parts. An in-depth microstructural analysis reveals that the competitive epitaxial growth between $<001>$ and $<101>$ ( viewed along the y-axis) columnar grains is crucial to the texture evolution in both the corner and central parts of the sample. On the contrary, an apparent crystallographic texture would not be formed if the ordered arrangement of temperature gradients in the AM process was interrupted by randomly distributed microstructural features such as spherical pores [35]. In this case, the thermal conditions are complicated with temperature gradients pointing towards various directions and hence epitaxial growth is hindered.

Different grain orientations and local microstructures provoke different mechanical properties [36]. The consistent texture from the bottom to the top and the expected similar other microstructural features, which were caused by similar thermal gradients during the $3 \mathrm{D}$ printing process, lead to the relatively homogeneous hardness distribution throughout the central part from the bottom to the top. On the other hand, the change of texture from the bottom to the top promotes the considerable hardness variation throughout the corner parts. Many other factors, including the more significant thermal gradients imposed by the adjacent ambient temperature field as discussed above, result in much higher densities of crystalline defects [11] and consequently the much higher hardness and hardness variation from the bottom to the top throughout the corners.

Materials properties are highly dependent on their microstructures. Different types of textures are preferred dependent on the applications. For example, epitaxial growth is important when highly textured or even single crystalline structures are preferred for high temperature engine applications because this would significantly improve the creep properties of materials $[37,38]$. On the other hand, small-sized equiaxed grains with random orientations are usually beneficial for strength and ductility at the ambient temperature.
Therefore, many researches have been carried out to achieve these kind of structures (e.g., Ref. [39] and [40]). Our study here provides guidance for achieving different types of texture for AM components.

\section{Conclusions}

In conclusion, the crystallographic texture at different parts of an LPBF CrMnFeCoNi HEA cube was characterized using EBSD. The texture evolution exhibited dissimilar behaviours at different locations within a single as-fabricated product. Such different texture developments produced by identical AM processing parameters are attributed to the disparity of local thermal histories between the central and corner parts. Through the comparison between the central and corner parts of the sample, it is found that the determination of favourable grain growth directions controlled by scan strategy at the initial stage may not persist congruously from the bottom to the top of the product. Subsequent interference to local thermal gradients originated from ambient temperature field could possibly surpass the supremacy of scan paths on determination of the $<001>$ texture horizontal growth directions. Epitaxial growth of the $<001>$ directions vertically between subsequent layers and horizontally between adjacent scan paths was an indispensable feature that facilitated the texture evolution. Hence, the texture difference contributes to the significant variations of mechanical properties within the as-built AM component.

\section{Acknowledgements}

The authors acknowledge the scientific and technical support from the Microscopy Australia node at the University of Sydney (Sydney Microscopy \& Microanalysis). This project is supported by the AustraliaUS Multidisciplinary University Research Initiative (AUSMURI) program. X.Z. Liao was also supported by the Australian Research Council [DP190102243], and S. Primig by [DE180100440].

\section{Funding}

Open Access funding enabled and organized by CAUL and its Member Institutions. 


\section{Declarations}

Conflict of interest The authors declare that they have no known competing financial interests or personal relationships that could have appeared to influence the work reported in this paper.

Open Access This article is licensed under a Creative Commons Attribution 4.0 International License, which permits use, sharing, adaptation, distribution and reproduction in any medium or format, as long as you give appropriate credit to the original author(s) and the source, provide a link to the Creative Commons licence, and indicate if changes were made. The images or other third party material in this article are included in the article's Creative Commons licence, unless indicated otherwise in a credit line to the material. If material is not included in the article's Creative Commons licence and your intended use is not permitted by statutory regulation or exceeds the permitted use, you will need to obtain permission directly from the copyright holder. To view a copy of this licence, visit http://creativecommons.org/licen ses/by $/ 4.0 /$.

\section{References}

[1] Herzog D, Seyda V, Wycisk E, Emmelmann C (2016) Additive manufacturing of metals. Acta Mater 117:371-392. https://doi.org/10.1016/j.actamat.2016.07.019

[2] Körner C (2016) Additive manufacturing of metallic components by selective electron beam melting - a review. Int Mater Rev 61(5):361-377. https://doi.org/10.1080/0950660 8.2016.1176289

[3] Gokuldoss PK, Kolla S, Eckert J (2017) Additive manufacturing processes: selective laser melting, electron beam melting and binder jetting-selection guidelines. Materials (Basel) 10(6):672. https://doi.org/10.3390/ma10060672

[4] Yap CY, Chua CK, Dong ZL, Liu ZH, Zhang DQ, Loh LE, Sing SL (2015) Review of selective laser melting: materials and applications. Appl Phys Rev 2(4):041101. https://doi. org/10.1063/1.4935926

[5] Zhang Y, Zuo TT, Tang Z, Gao MC, Dahmen KA, Liaw PK, Lu ZP (2014) Microstructures and properties of high-entropy alloys. Prog Mater Sci 61:1-93. https://doi.org/10.1016/j.p matsci.2013.10.001

[6] Wang YM, Voisin T, McKeown JT, Ye J, Calta NP, Li Z, Zeng Z, Zhang Y, Chen W, Roehling TT, Ott RT, Santala MK, Depond PJ, Matthews MJ, Hamza AV, Zhu T (2018) Additively manufactured hierarchical stainless steels with high strength and ductility. Nat Mater 17(1):63-71. https://d oi.org/10.1038/nmat5021

[7] Song M, Zhou R, Gu J, Wang Z, Ni S, Liu Y (2020) Nitrogen induced heterogeneous structures overcome strength-ductility trade-off in an additively manufactured high-entropy alloy. Appl Mater Today 18:100498. https://d oi.org/10.1016/j.apmt.2019.100498

[8] Zhu ZG, An XH, Lu WJ, Li ZM, Ng FL, Liao XZ, Ramamurty U, Nai SML, Wei J (2019) Selective laser melting enabling the hierarchically heterogeneous microstructure and excellent mechanical properties in an interstitial solute strengthened high entropy alloy. Mater Res Lett 7(11):453-459. https://doi.org/10.1080/21663831.2019. 1650131

[9] Ma E, Zhu T (2017) Towards strength-ductility synergy through the design of heterogeneous nanostructures in metals. Mater Today 20(6):323-331. https://doi.org/10.1016/j. mattod.2017.02.003

[10] Haghdadi N, Laleh M, Moyle M, Primig S (2021) Additive manufacturing of steels: a review of achievements and challenges. J Mater Sci 56(1):64-107. https://doi.org/10.10 07/s10853-020-05109-0

[11] Wang H, Zhu ZG, Chen H, Wang AG, Liu JQ, Liu HW, Zheng RK, Nai SML, Primig S, Babu SS, Ringer SP, Liao XZ (2020) Effect of cyclic rapid thermal loadings on the microstructural evolution of a $\mathrm{CrMnFeCoNi}$ high-entropy alloy manufactured by selective laser melting. Acta Mater 196:609-625. https://doi.org/10.1016/j.actamat.2020.07.006

[12] Wang H, Chen D, An X, Zhang Y, Sun S, Tian Y, Zhang Z, Wang A, Liu J, Song M (2021) Deformation-induced crystalline-to-amorphous phase transformation in a CrMnFeCoNi high-entropy alloy. Sci Adv 7:eabe3105. https://doi. org/10.1126/sciadv.abe3105

[13] Zhu ZG, Nguyen QB, Ng FL, An XH, Liao XZ, Liaw PK, Nai SML, Wei J (2018) Hierarchical microstructure and strengthening mechanisms of a CoCrFeNiMn high entropy alloy additively manufactured by selective laser melting. Scripta Mater 154:20-24. https://doi.org/10.1016/j.scriptama t.2018.05.015

[14] Manvatkar V, De A, DebRoy T (2014) Heat transfer and material flow during laser assisted multi-layer additive manufacturing. J Appl Phys 116(12):124905. https://doi.org/ $10.1063 / 1.4896751$

[15] Basak A, Das S (2016) Epitaxy and microstructure evolution in metal additive manufacturing. Annu Rev Mater Res 46(1):125-149. https://doi.org/10.1146/annurev-matsci-070 $115-031728$

[16] Wei HL, Mazumder J, DebRoy T (2015) Evolution of solidification texture during additive manufacturing. Sci Rep 5:16446. https://doi.org/10.1038/srep16446 
[17] Fredriksson H, Åkerlind U (2012) Solidification and crystallization processing in metals and alloys. John Wiley \& Sons, Hoboken

[18] Stephenson P, Haghdadi N, DeMott R, Liao X, Ringer S, Primig S (2020) Effect of scanning strategy on variant selection in additively manufactured Ti-6Al-4V. Addit Manuf 36:101581. https://doi.org/10.1016/j.addma.2020. 101581

[19] Sun S-H, Hagihara K, Nakano T (2018) Effect of scanning strategy on texture formation in $\mathrm{Ni}-25$ at.\%Mo alloys fabricated by selective laser melting. Mater Des 140:307-316. h ttps://doi.org/10.1016/j.matdes.2017.11.060

[20] Marattukalam JJ, Karlsson D, Pacheco V, Beran P, Wiklund U, Jansson U, Hjörvarsson B, Sahlberg M (2020) The effect of laser scanning strategies on texture, mechanical properties, and site-specific grain orientation in selective laser melted 316L SS. Mater Des 193:108852. https://doi.org/10. 1016/j.matdes.2020.108852

[21] Geiger F, Kunze K, Etter T (2016) Tailoring the texture of IN738LC processed by selective laser melting (SLM) by specific scanning strategies. Mater Sci Eng, A 661:240-246. https://doi.org/10.1016/j.msea.2016.03.036

[22] Haghdadi N, Primig S, Annasamy M, Cizek P, Hodgson P, Fabijanic D (2020) On the hot-worked microstructure of a facecentered cubic A10. 3CoCrFeNi high entropy alloy. Scr Mater 178:144-149. https://doi.org/10.1016/j.scriptamat.2019.11.022

[23] Wang P, Ma L, Cheng X, Li X (2021) Effect of grain size and crystallographic orientation on the corrosion behaviors of low alloy steel. J Alloy Compd 857:158258. https://doi. org/10.1016/j.jallcom.2020.158258

[24] Ishimoto T, Hagihara K, Hisamoto K, Sun S-H, Nakano T (2017) Crystallographic texture control of beta-type Ti$15 \mathrm{Mo}-5 \mathrm{Zr}-3 \mathrm{Al}$ alloy by selective laser melting for the development of novel implants with a biocompatible low Young's modulus. Scr Mater 132:34-38. https://doi.org/10. 1016/j.scriptamat.2016.12.038

[25] Arias-González F, del Val J, Comesaña R, Penide J, Lusquiños F, Quintero F, Riveiro A, Boutinguiza M, Gil FJ, Pou J (2018) Microstructure and crystallographic texture of pure titanium parts generated by laser additive manufacturing. Met Mater Int 24(1):231-239. https://doi.org/10.1007/s125 40-017-7094-x

[26] Hagihara K, Nakano T, Suzuki M, Ishimoto T, Suyalatu S-H (2017) Successful additive manufacturing of MoSi2 including crystallographic texture and shape control. J Alloy Compd 696:67-72. https://doi.org/10.1016/j.jallcom.2016.1 1.191

[27] DebRoy T, David SA (1995) Physical processes in fusion welding. Rev Mod Phys 67(1):85-112. https://doi.org/10.1 103/RevModPhys.67.85
[28] Blecher JJ, Palmer TA, DebRoy T (2013) Solidification map of a nickel-base alloy. Metall and Mater Trans A 45(4):2142-2151. https://doi.org/10.1007/s11661-013-21491

[29] Dinda GP, Dasgupta AK, Mazumder J (2012) Texture control during laser deposition of nickel-based superalloy. Scr Mater 67(5):503-506. https://doi.org/10.1016/j.scriptamat.2 012.06 .014

[30] Gludovatz B, Hohenwarter A, Catoor D, Chang EH, George EP, Ritchie RO (2014) A fracture-resistant high-entropy alloy for cryogenic applications. Science 345(6201):1153-1158. https://doi.org/10.1126/science. 1254581

[31] Dinda GP, Dasgupta AK, Mazumder J (2009) Laser aided direct metal deposition of Inconel 625 superalloy: microstructural evolution and thermal stability. Mater Sci Eng, A 509(1-2):98-104. https://doi.org/10.1016/j.msea.20 09.01.009

[32] Fernandez-Zelaia P, Kirka MM, Rossy AM, Lee Y, Dryepondt SN (2021) Nickel-based superalloy single crystals fabricated via electron beam melting. Acta Mater 216:117133. https://doi.org/10.1016/j.actamat.2021.117133

[33] Liu P, Ji Y, Wang Z, Qiu C, Antonysamy A, Chen L-Q, Cui $X$, Chen L (2018) Investigation on evolution mechanisms of site-specific grain structures during metal additive manufacturing. J Mater Process Technol 257:191-202

[34] Sofinowski KA, Raman S, Wang X, Gaskey B, Seita M (2021) Layer-wise engineering of grain orientation (LEGO) in laser powder bed fusion of stainless steel 316L. Addit Manuf 38:101809. https://doi.org/10.1016/j.addma.2020. 101809

[35] Kenney MJ, O’Donnell K, Quintana MJ, Collins PC (2021) Spherical pores as 'microstructural informants': understanding compositional, thermal, and mechanical gyrations in additively manufactured Ti-6Al-4V. Scr Mater 198:113827. https://doi.org/10.1016/j.scriptamat.2021. 113827

[36] Yu C-H, Peng RL, Luzin V, Sprengel M, Calmunger M, Lundgren J-E, Brodin H, Kromm A, Moverare J (2020) Thin-wall effects and anisotropic deformation mechanisms of an additively manufactured Ni-based superalloy. Addit Manuf 36:101672

[37] Cervellon A, Hémery S, Kürnsteiner P, Gault B, Kontis P, Cormier J (2020) Crack initiation mechanisms during very high cycle fatigue of Ni-based single crystal superalloys at high temperature. Acta Mater 188:131-144. https://doi.org/ 10.1016/j.actamat.2020.02.012

[38] Reed RC (2008) The superalloys: fundamentals and applications. Cambridge University Press, Cambridge 
[39] Todaro C, Easton M, Qiu D, Zhang D, Bermingham M, Lui E, Brandt M, StJohn D, Qian M (2020) Grain structure control during metal 3D printing by high-intensity ultrasound. Nat Commun 11(1):1-9. https://doi.org/10.1038/s41 467-019-13874-Z

[40] Xu W, Lui EW, Pateras A, Qian M, Brandt M (2017) In situ tailoring microstructure in additively manufactured Ti-6Al-
$4 \mathrm{~V}$ for superior mechanical performance. Acta Mater 125:390-400. https://doi.org/10.1016/j.actamat.2016.12.027

Publisher's Note Springer Nature remains neutral with regard to jurisdictional claims in published maps and institutional affiliations. 